\title{
ISOGEOMETRIC ANALYSIS OF THE UV IRRADIATION CURING PROCESS IN ADDITIVE MANUFACTURING
}

\author{
Maged Shaban ${ }^{1,3}$, Clemens Verhoosel ${ }^{1}$, Herman Wijshoff $^{2}$, Alessandro Reali $^{3}$, \\ Ferdinando Auricchio ${ }^{3}$ and Harald van Brummelen ${ }^{1}$ \\ ${ }^{1}$ Eindhoven University of Technology \\ P.O. Box 513, 5600 MB Eindhoven, The Netherlands \\ e-mail:m.m.m.shaban@tue.nl, c.v.verhoosel@tue.nl,e.h.v.brummelen@tue.nl \\ ${ }^{2}$ Canon Production Printing Holding B.V. \\ P.O. Box 101, 5900 MA, Venlo, The Netherlands \\ e-mail: herman.wijshoff@cpp.canon \\ ${ }^{3}$ University of Pavia, 27100 Pavia, Italy \\ e-mail: alessandro.reali@unipv.it, auricchi@unipv.it
}

Key Words: Additive manufacturing, 3D printing, Photopolymerization, Isogeometric analysis.

The photopolymerization-based additive manufacturing process, including 3D printing techniques such as stereolithography and digital light processing, has undergone considerable development over the last few decades. These techniques provide flexibility in the physical and chemical properties of 3D printed materials [1]. The photopolymerization process is based on ultraviolet (UV) curing of liquidstate monomers/oligomers in the presence of photopolymerizable photoinitiators [2], which is known as the UV irradiation curing process.

The UV light intensity has a significant effect on the reactivity and solidification process, modeling which has many challenges. In this work, we present a novel model that determines the required UV light intensity in the photopolymerization process. To make meaningful predictions of the UV light intensity influence on the curing process, it is essential to appropriately model the UV light waves. Maxwell's equations are considered for this purpose. The photopolymerization process itself is described by the reaction-diffusion equation, which is coupled to Maxwell's equations in a unit square of the resin. Isogeometric analysis is used to discretize the produced coupled system of equations [3]. We present numerical results which demonstrate the light intensity influence on the UV curing process.

\section{REFERENCES}

[1] Crivello, J. V., \& Reichmanis, E. (2014). Photopolymer materials and processes for advanced technologies. Chemistry of Materials, Vol. 26, pp. 533-548.

[2] Westbeek, S., van Dommelen, J. A. W., Remmers, J. J. C., \& Geers, M. G. D. (2018). Multiphysical modeling of the photopolymerization process for additive manufacturing of ceramics. European Journal of Mechanics, A/Solids, 71, 210-223.

[3] Buffa, A., Sangalli, G., \& Vázquez, R. (2014). Isogeometric methods for computational electromagnetics: B-spline and T-spline discretizations. Journal of Computational Physics, 257(PB), 129. 